\title{
STRONG PROXIMINALITY AND POLYHEDRAL SPACES
}

\author{
G. GODEFROY and V. INDUMATHI
}

\begin{abstract}
In any dual space $X^{*}$, the set QP of quasi-polyhedral points is contained in the set SSD of points of strong subdifferentiability of the norm which is itself contained in the set NA of norm attaining functionals. We show that NA and SSD coincide if and only if every proximinal hyperplane of $X$ is strongly proximinal, and that if QP and NA coincide then every finite codimensional proximinal subspace of $X$ is strongly proximinal. Natural examples and applications are provided.
\end{abstract}

\section{Introduction}

Let $X$ be a Banach space and $Y$ be a finite codimensional closed subspace of $X$. Pick a vector $x \in X$ and $\epsilon>0$. It follows from the definition of the distance $d(x, Y)$ from $x$ to $Y$ that there exists $y \in Y$ such that $\|x-y\| \leq$ $d(x, Y)+\epsilon$. When is it so that such a "nearly best approximant" of $x$ in $Y$ is necessarily close to an actual best approximant? If this takes place for every $x \in X$, the space $Y$ has to be proximinal, but the converse fails. Let us say that $Y$ is strongly proximinal if this condition holds (definition 2.1). We study in this work the class of Banach spaces for which every finite codimensional proximinal subspace is strongly proximinal. Quite surprisingly, this class turns out to contain many natural examples of spaces which are non-reflexive and non strictly convex, and among them certain polyhedral spaces. Good selections of metric projections (that is, first Baire class or continuous ones) are also investigated.

We now turn to a detailed description of our results. Section 1 is devoted to strong sub-differentiability (in short, S.S.D.) in dual spaces. Lemma 1.1. explains how to find S.S.D. points of $X^{*}$ through the predual $X$. The more technical section 2 provides a characterization (Theorem

2000 Mathematics Subject Classification: 46B20.

Servicio de Publicaciones. Universidad Complutense. Madrid, 2001 
2.5) of finite codimensional strongly proximinal subspaces among proximinal ones. It follows for instance that if $X$ is reflexive and $f \in X^{*}$ is a point of Gâteaux smoothness but not of Fréchet smoothness, then the corresponding hyperplane is not strongly proximinal. Spaces in which every proximinal hyperplane is strongly proximinal are characterized (Proposition 2.6). Quasi-polyhedral points (in short, (QP)-points) in dual spaces are used in section 3 to formulate a sufficient condition for the equivalence between proximinality and its strong version, which is our main result (Theorem 3.4). The proof relies on the results from sections 1 and 2. A recent result of Fonf and Lindenstrauss ([F-L]) shows that this theorem applies in particular to every subspace of a polyhedral predual of $l^{1}(\mathbf{N})$. Section 4 is devoted to selectors of the metric projection. Jayne-Rogers selection theorem shows that a first Baire class selector exists when strong proximinality holds (Proposition 4.2) while Michael's theorem shows through a different argument that a continuous selector exists when the orthogonal space is polyhedral (Proposition 4.5). Therefore, continuous selectors exist under the assumptions of Theorem 3.4.

\section{Notations.}

We denote by $X$ a real normed linear space and $X^{*}$ its dual. The closed unit ball of $X,\{x \in X:\|x\| \leq 1\}$, is denoted by $B_{X}$ and the unit sphere, $\{x \in X:\|x\|=1\}$, by $S_{X}$. If $Y$ is a closed subspace of $X$, the annihilator space $Y^{\perp}$ is given by

$$
Y^{\perp}=\left\{f \in X^{*}: f(y)=0 \forall y \in Y\right\} .
$$

If $A$ is a closed subset of $X$ and $x \in X, d(x, A)$ denotes the distance $\inf _{a \in A}\|x-a\|$ of $x$ from the set $A$. For $x \in X$, the set of nearest points to $x$ from $Y$ is given by

$$
P_{Y}(x)=\{y \in Y:\|x-y\|=d(x, Y)\}
$$

and for $\epsilon>0$, we set

$$
P_{Y}(x, \epsilon)=\{y \in Y:\|x-y\|<d(x, Y)+\epsilon\} .
$$

Subspaces are always assumed to be closed in the norm topology. We recall that a subspace $Y$ of $X$ is said to be proximinal if $P_{Y}(x) \neq \emptyset$ for all $x \in X$. 
For $x \in X$ and $f \in X^{*}$, we set

$$
J_{X^{*}}(x)=\left\{f \in S_{X^{*}}: f(x)=\|x\|\right\}
$$

and

$$
J_{X}(f)=\left\{x \in S_{X}: f(x)=\|f\|\right\}
$$

We say $f \in S_{X^{*}}$ is in $N A_{1}$ if $J_{X}(f) \neq \emptyset$, and we define

$$
N A=\{0\} \cup\left\{f \in X^{*}: \frac{f}{\|f\|} \in N A_{1}\right\}
$$

It is clear that $N A$ coincide with the set of all elements of $X^{*}$ which attain their norm. A subset $B$ of $S_{X^{*}}$ is called a boundary for $X$ if for all $x \in S_{X}, B \cap J_{X^{*}}(x) \neq \emptyset$. We denote by $B^{\prime}$ the set of weak* accumulation points of $B$.

The following notations will be used in section 2. Given a set of linearly independent functionals $f_{1}, \cdots, f_{n}$ of $X^{*}$, we define the following sets and numbers as in [I]. Having defined $J_{X}\left(f_{1}\right)$ by (1), we inductively define for $2 \leq i \leq n$,

$$
J_{X}\left(f_{1}, f_{2} \cdots f_{i}\right)=\left\{x \in J_{X}\left(f_{1}, \cdots f_{i-1}\right): f_{i}(x)=\sup _{x^{\prime} \in J_{X}\left(f_{1} \cdots f_{i-1}\right)} f_{i}\left(x^{\prime}\right)\right\}
$$

We set

$$
M\left(f_{1}\right)=\left\|f_{1}\right\|=\tilde{N}\left(f_{1}\right)
$$

and for $\epsilon>0$,

$$
J_{X}\left(f_{1}, \epsilon\right)=\left\{x \in B_{X}: f_{1}(x)>\left\|f_{1}\right\|-\epsilon\right\}
$$

Again, for $2 \leq i \leq n$, we inductively define

$$
\begin{gathered}
M\left(f_{1} \cdots f_{i}\right)=\sup \left\{f_{i}(x): x \in J_{X}\left(f_{1} \cdots f_{i-1}\right)\right\} \\
\tilde{N}\left(f_{1} \cdots f_{i}, \epsilon\right)=\sup \left\{f_{i}(x): x \in J_{X}\left(f_{1} \cdots f_{i-1}, \epsilon\right)\right\} \\
\tilde{N}\left(f_{1} \cdots f_{i}\right)=\inf _{\epsilon>0} \tilde{N}\left(f_{1} \cdots f_{i}, \epsilon\right)
\end{gathered}
$$

and finally

$$
J_{X}\left(f_{1} \cdots f_{i}, \epsilon\right)=\left\{x \in J_{X}\left(f_{1} \cdots f_{i-1}, \epsilon\right): f_{i}(x)>\tilde{N}\left(f_{1} \cdots f_{i}\right)-\epsilon\right\}
$$

Other notations are classical and can be found e.g. in [L-T]. 


\section{Strong sub-differentiability in dual spaces}

Let $X$ be a Banach space. A norm $\|$. $\|$ on $X$ is said to be strongly sub-differentiable (SSD) at $x$ (See $[\mathrm{F}-\mathrm{P}],[\mathrm{G}-\mathrm{M}-\mathrm{Z}])$ if the one-sided limit

$$
\lim _{t \rightarrow 0^{+}} 1 / t(\|x+t h\|-\|x\|)
$$

exists uniformly in $h \in S_{X}$. In theorem 3.3 from [F-P], it is shown that

$$
f \in S_{X^{*}},\|\cdot\|_{X^{*}} \mathrm{SSD} \text { at } f \Rightarrow f \in N A_{1} .
$$

In fact, more is true. The following Lemma provides a crucial link between the strong sub-differentiability of dual norms and strong proximinality, as defined in Section 2 below.

Lemma 1.1. Let $X$ be a Banach space and $f \in S_{X^{*}}$. Then the following are equivalent.

1. The dual norm $\|\cdot\|_{X^{*}}$ is SSD at $f$.

2. We have $f \in N A_{1}$ and for all $\epsilon>0$, there exists $\delta>0$ such that

$$
f(x)>1-\delta \Rightarrow d\left(x, J_{X}(f)\right)<\epsilon .
$$

Proof. First we show that 2) implies 1). In Theorem 1.2 of $[\mathrm{F}-\mathrm{P}]$ it is shown that $\|\cdot\|_{X^{*}}$ is SSD at $f$ if and only if given $\epsilon>0$, there exists $\delta>0$ such that

$$
y \in B_{X^{* *}}, y(f)>1-\delta \Rightarrow d\left(y, J_{X^{* *}}(f)\right)<\epsilon .
$$

If 1$)$ does not hold then there is a sequence $\left(y_{n}\right) \subseteq B_{X^{* *}}$ and $\epsilon>0$ such that $\lim _{n \rightarrow \infty} y_{n}(f)=1$ but $y_{n} \notin C_{\epsilon}$ for all $n$, where $C_{\epsilon}=J_{X^{* *}}(f)+$ $\epsilon B_{X^{* *}}$. Now the set $C_{\epsilon}$ is weak* compact and so is weak*closed. Now using weak* density of $B_{X}$ in $B_{X^{* *}}$, we get a sequence $x_{n} \in B_{X} \cap\left(X^{* *} \backslash C_{\epsilon}\right)$ for all $n$ and $\lim _{n \rightarrow \infty} f\left(x_{n}\right)=1$. Clearly this contradicts 2$)$.

We now prove that 1) implies 2). Given $\epsilon>0$ let $\delta>0$ be such that

$$
y \in B_{X^{* *}}, y(f)>1-\delta \Rightarrow d\left(y, J_{X^{* *}}(f)\right)<\frac{\epsilon}{2} .
$$

Select any $x \in B_{X}$ with $f(x)>1-\delta$ and $t \in J_{X^{* *}}(f)$ with $\|x-t\|<\frac{\epsilon}{2}$. Pick $\left(x_{\alpha}\right) \subset B_{X}$ with $\left(x_{\alpha}\right)$ converging weak* to $t$. 
Fact. For any $\delta_{1}>0$, there exists $c \in \operatorname{conv}\left(x_{\alpha}\right) \subset B_{X}$ with $\|x-c\|<\frac{\epsilon}{2}$ and $f(c)>1-\delta_{1}$.

Indeed, without loss of generality, $f\left(x_{\alpha}\right)>1-\delta_{1}$ for all $\alpha$. If $\|x-c\| \geq \frac{\epsilon}{2}$ for all $c \in \operatorname{conv}\left(x_{\alpha}\right)$, there exists $h \in B_{X^{*}}$ such that if $c \in$ $\operatorname{conv}\left(x_{\alpha}\right)$,

$$
h(x-c) \geq \frac{1}{2}\left(\|x-t\|+\frac{\epsilon}{2}\right)
$$

and thus $h(x-t)>\|x-t\|$ which gives a contradiction.

Choosing properly $\delta_{1}>0$ we find $x_{1} \in B_{X}$ such that $\left\|x-x_{1}\right\|<\frac{\epsilon}{2}$ and $d\left(x_{1}, J_{X^{* *}}(f)\right)<\frac{\epsilon}{4}$.

Following the same steps, we construct inductively a sequence $\left(x_{n}\right)_{n \geq 1}$ in $B_{X}$ such that

$$
\left\|x_{n}-x_{n+1}\right\|<\frac{\epsilon}{2^{n+1}} ; d\left(x_{n}, J_{X^{* *}}(f)\right)<\frac{\epsilon}{2^{n+1}}
$$

Clearly

$$
x_{\infty}=\lim _{n \rightarrow \infty} x_{n} \in X \cap J_{X^{* *}}(f)=J_{X}(f)
$$

and $\left\|x-x_{\infty}\right\|<\epsilon$.

Remarks 1.2. 1) It is clear from the above proof that for every $x \in B_{X}$, $d\left(x, J_{X^{* *}}(f)\right)=d\left(x, J_{X}(f)\right)$.

2) It is easy to see that

$$
{\overline{J_{X}}}^{w *}(f) \subseteq J_{X^{* *}}(f)
$$

In general, this inclusion is strict even if $f \in N A_{1}$ : consider for instance the space $X=\left(l_{1}(\mathbb{N}),\|\cdot\|_{1}\right)$ and $f=\left(1,\left(1-\frac{1}{n}\right)_{n \geq 2}\right) \in X^{*}$. However if $\|\cdot\|_{X^{*}}$ is $\operatorname{SSD}$ at $f$, then

$$
{\overline{J_{X}}}^{w *}(f)=J_{X^{* *}}(f)
$$

Indeed, pick $t \in J_{X^{* *}}(f)$. There is $\left(x_{\alpha}\right) \subseteq B_{X}$ such that $t=w^{*} \lim \left(x_{\alpha}\right)$. Since $\lim f\left(x_{\alpha}\right)=1$ there is $y_{\alpha} \in J_{X}(f)$ such that $\lim \left\|x_{\alpha}-y_{\alpha}\right\|=0$, and then $t=w^{*} \lim \left(y_{\alpha}\right)$.

3) When a dual norm is $S S D$ at $f$, then in general $J_{X}(f)$ is a strict subset of $J_{X^{* *}}(f)$, and thus Lemma 1.1 is not a consequence of Theorem 1.2 in $[\mathrm{F}-\mathrm{P}]$. A simple example is provided by any $f \in l^{1}$ with finite support (see $[\mathrm{G}-\mathrm{I}]$ ). 


\section{Strong proximinality}

The following terminology is motivated by the well-known notion of "strong minimum" in optimization theory. To the best of our knowledge, the corresponding notion of "strong proximinality" has not been given a name so far. Hence we state:

Definition 2.1. Let $X$ be a Banach space and $Y$ be a proximinal subspace of $X$. Then $Y$ is said to be strongly proximinal if for every $x \in X$ and every $\delta>0$, there is $\epsilon>0$ such that $d\left(y, P_{Y}(x)\right)<\delta$ for all $y \in P_{Y}(x, \epsilon)$.

Remark 2.2. Ka Sing Lau $[\mathrm{K}]$ defined the notion of "local uniform proximinality" as follows. For a closed subspace $Y$ of a normed linear space $X$ and $x \in X$, set $Y_{x}=Y+s p(x)$ and for $\epsilon>0$ denote

$$
\alpha(x, \epsilon)=\inf \left\{r>0:(1+\epsilon) B_{X} \cap\left(B_{Y_{x}}+Y\right) \subseteq B_{X}+r B_{Y}\right\} .
$$

Then the subspace $Y$ is said to be locally $\mathrm{U}$-proximinal if for each $x \in X$, one has $\lim _{\epsilon \rightarrow 0} \alpha(x, \epsilon)=0$. It is shown in $[\mathrm{K}]$ that locally $\mathrm{U}$-proximinal subspaces are proximinal. It is easily verified that for $x \in X$ with $d(x, Y)=1$ and any $\epsilon>0$,

$$
\sup \left\{d\left(y, P_{Y}(x)\right): y \in P_{Y}(x, \epsilon)\right\} \leq \alpha(x, \epsilon)
$$

and therefore local U-proximinality implies strong proximinality. We do not have a example showing that the converse implication fails.

We now proceed to find conditions for strong proximinality. We use the following notation. Let $Y$ be a proximinal subspace of $X$, let $x \in X$ and $\epsilon>0$. We denote

$$
Q_{Y}(x)=x-P_{Y}(x) ; Q_{Y}(x, \epsilon)=x-P_{Y}(x, \epsilon)
$$

and

$$
R_{Y}(x, \epsilon)=\frac{1}{1+\epsilon} Q_{Y}(x, \epsilon)
$$

It is easy to see that if $Y$ is proximinal then with this notation one has

$\mathrm{Y}$ is strongly proximinal

$$
\begin{aligned}
& \Leftrightarrow \lim _{\epsilon \rightarrow 0} \sup \left\{d\left(y, P_{Y}(x)\right): y \in P_{Y}(x, \epsilon)\right\}=0 \\
& \Leftrightarrow \lim _{\epsilon \rightarrow 0} \sup \left\{d\left(y, Q_{Y}(x)\right): y \in Q_{Y}(x, \epsilon)\right\}=0 \\
& \Leftrightarrow \lim _{\epsilon \rightarrow 0} \sup \left\{d\left(y, Q_{Y}(x)\right): y \in R_{Y}(x, \epsilon)\right\}=0
\end{aligned}
$$


and each of the last three conditions in the above equivalence hold for all $x \in X$ if it holds for every $x \in X$ with $\|x\|=1=d(x, Y)$.

If $x \in X$ satisfies $\|x\|=1=d(x, Y)$ then

$$
\begin{aligned}
& Q_{Y}(x)=\left\{y \in X:\|y\|=1 \text { and } f(y)=f(x) \forall f \in Y^{\perp}\right\} \\
& Q_{Y}(x, \epsilon)=\left\{y \in X:\|y\| \leq 1+\epsilon \text { and } f(y)=f(x) \forall f \in Y^{\perp}\right\}
\end{aligned}
$$

and therefore

$$
R_{Y}(x, \epsilon) \subseteq\left\{y \in B_{X}:|f(y)-f(x)|<\epsilon \quad \forall f \in B_{Y^{\perp}}\right\}
$$

Let us gather these observations in the following

Fact. A proximinal subspace $Y$ of finite codimension in $X$ is strongly proximinal if and only if for any $x \in X$ with $\|x\|=1=d(x, Y)$ and $\epsilon>0$, there exists $\delta>0$ such that if $y \in B_{X}$ and $|f(y)-f(x)|<\delta$ for all $f \in B_{Y^{\perp}}$, then there exists $z \in B_{X}$ with $x-z \in Y$ and $\|y-z\|<\epsilon$.

Clearly sufficiency follows easily from (2) and (3). To see the necessity, we observe that given $\epsilon>0$, by (2), there exists $\eta>0$ such that $\eta<\frac{\epsilon}{2}$ and

$$
v \in Q_{Y}(x, \eta) \Rightarrow d\left(v, Q_{Y}(x)\right)<\frac{\epsilon}{2} .
$$

Now since $(X / Y)^{*}$ is isometric to $Y^{\perp}$, we find $\delta>0$ such that if $y \in B_{X}$ and $|f(y)-f(x)|<\delta$ for all $f \in B_{Y^{\perp}}$, then there exists $w \in X$ with $\|w\|<\eta$ and $f(w)=f(x-y)$ for all $f \in Y^{\perp}$.

Then $v=y+w \in Q_{Y}(x, \eta)$ and (4) concludes the proof of the Fact. Now a formal verification provides the following equivalent formulations of strong proximinality for a proximinal subspace $Y$ of finite codimension $n$.

Proposition 2.3. Let $X$ be a Banach space and $Y$ be a proximinal subspace of finite codimension $n$ in $X$. Then the following are equivalent:

1. $Y$ is strongly proximinal.

2. For any $x \in X$ with $\|x\|=1=d(x, Y)$ and every basis $f_{1}, \cdots, f_{n}$ of $Y^{\perp}$, given $\epsilon>0$, there exists $\delta>0$ such that if $y \in B_{X}$ and $\left|f_{i}(y)-f_{i}(x)\right|<\delta$ for $1 \leq i \leq n$, then there exists $z \in B_{X}$ with

$$
x-z \in Y \text { and }\|y-z\|<\epsilon \text {. }
$$


3. For any $x \in X$ with $\|x\|=1=d(x, Y)$, there is a basis $f_{1}, \cdots, f_{n}$ of $Y^{\perp}$ such that given $\epsilon>0$, there exists $\delta>0$ for which (5) holds.

4. For any $x \in X$ with $\|x\|=1=d(x, Y)$ and $\epsilon>0$, there exists $\delta>0$ such that if $y \in B_{X}$ and $\|x-y+Y\|<\delta$, then there exists $z \in B_{X}$ with $x-z \in Y$ and $\|y-z\|<\epsilon$.

We now proceed to derive a simpler characterization of strongly proximinal subspaces of finite codimension. We first recall a characterization of proximinal subspaces of finite codimension from [I], where a slightly different notation is used.

Theorem [I]. Let $X$ be a normed linear space and $Y$ be a closed subspace of codimension $n$ in $X$. Then $Y$ is proximinal in $X$ if and only if for every basis $f_{1}, \cdots, f_{n}$ of $Y^{\perp}$

1. $J_{X}\left(f_{1}, \cdots, f_{i}\right) \neq \emptyset$ for $1 \leq i \leq n$.

2. $\tilde{N}\left(f_{1}, \cdots, f_{i}\right)=M\left(f_{1}, \cdots, f_{i}\right)$ for $1 \leq i \leq n$.

We now seek extra conditions under which a proximinal subspace of finite codimension becomes strongly proximinal, and thus under which minimizing sequences of "approximate" nearest points can be used for locating actual nearest points. This querry will eventually lead us to our main result (Theorem 3.4).

If $x \in B_{X}$ with $\left.x\right|_{Y^{\perp}}$ in $\operatorname{ext}\left(S_{\left(Y^{\perp}\right)^{*}}\right)$, then $x \in J_{X}\left(f_{1}, \cdots, f_{n}\right)$ for a suitable basis $f_{1}, \cdots, f_{n}$ of $Y^{\perp}$, hence $\left\{\left.x\right|_{Y^{\perp}}\right\}=\left.J_{X}\left(f_{1}, \cdots, f_{n}\right)\right|_{Y^{\perp}}$. In this case, if

$$
\lim _{\epsilon \rightarrow 0} \sup \left\{d\left(y, J_{X}\left(f_{1}, \cdots, f_{n}\right)\right): y \in J_{X}\left(f_{1}, \cdots, f_{n}, \epsilon\right)\right\}=0
$$

then clearly condition 3) of Proposition 2.3 holds for $x$. If $\left.x\right|_{Y^{\perp}}$ is not in $\operatorname{ext}\left(S_{\left(Y^{\perp}\right)^{*}}\right)$,we need an alternate condition to replace " $x \in J_{X}\left(f_{1}, \cdots, f_{n}\right)$ " and hence we need the following result. It is very likely that this simple proposition is already known.

Proposition 2.4. Let $E$ be a n-dimensional normed linear space and $x_{0} \in S_{E} \backslash \operatorname{ext}\left(B_{E}\right)$. Let $F$ be the minimal extremal subset of $S_{E}$ to which $x_{0}$ belongs. If $F=J_{E}\left(f_{1}, \cdots, f_{l}\right), l<n$, for a suitable set of linearly independent functionals $f_{1}, \cdots, f_{l}$ of $E^{*}$, the set $f_{1}, \cdots, f_{l}$ can be extended to a basis $f_{1}, \cdots, f_{n}$ of $E^{*}$ such that 


$$
\begin{array}{r}
\inf \left\{f_{i}(x): f_{j}\left(x_{0}\right)=f_{j}(x) \text { for } 1 \leq j \leq i-1\right\}<f_{i}\left(x_{0}\right)< \\
\sup \left\{f_{i}(x): f_{j}\left(x_{0}\right)=f_{j}(x) \text { for } 1 \leq j \leq i-1\right\} \text { for } i=l+1, \cdots, n .
\end{array}
$$

Proof. Choose any $f_{l+1} \in E^{*}$ such that $f_{1}, \cdots, f_{l+1}$ is a linearly independent subset of $E^{*}$. Clearly, by assumption, $x_{0} \notin J_{X}\left(f_{1}, \cdots, f_{l+1}\right)$ and $x_{0} \notin J_{X}\left(f_{1}, \cdots,-f_{l+1}\right)$. Thus (6) is satisfied for $i=l+1$. Assume inductively that (6) is satisfied up to $i$, for some $i, l+1 \leq i \leq n$. If $i=n$ there is nothing to prove. Assume $l+1 \leq i<n$. We now proceed to get $f_{i+1} \in E^{*}$ so that $f_{1}, \cdots, f_{i+1}$ is a linearly independent set and (6) holds when $i$ is replaced by $i+1$.

Let r.bd and r.int denote relative boundary and relative interior respectively. Set

$$
L=\cap_{j=1}^{i}\left\{x \in E: f_{j}(x)=f_{j}\left(x_{0}\right)\right\} .
$$

Then $L=H+x_{0}$ where $H=\cap_{j=1}^{i} \operatorname{Kerf}_{j}$. We have $L \cap \operatorname{r.bd} F \neq \emptyset$. Pick any $z \in L \cap \operatorname{r.bd} F$. Then $z \in S_{E}$ and by theorem 18.2 in [R], $z \in \operatorname{rint} G$, where $G$ is a face of $B_{E}$. Also, $z \in \operatorname{rod} F \subseteq F$ and $F$ is extremal. Hence $G$ is a proper subset $F$ and $\operatorname{dim} G<\operatorname{dim} F$. So, there exists $f_{i+1} \in E^{*}$ such that $J_{X}\left(f_{1}, \ldots, f_{l}, f_{i+1}\right)$ is a proper subset of $F$ and $G \subseteq J_{X}\left(f_{1}, \ldots, f_{l}, f_{i+1}\right)$. Clearly

$$
f_{i+1}\left(x_{0}\right)<\sup _{x \in F \cap L} f_{i+1}(x)=f_{i+1}(z)
$$

as $F$ is the minimal face to which $x_{0}$ belongs. With $z \in L$, the above inequality implies that the functionals $f_{1}, \cdots, f_{i}, f_{i+1}$ form a linearly independent set.

We now verify that $f_{i+1}\left(x_{0}\right)>\inf _{y \in F \cap L} f_{i+1}(y)$. We have $x_{0} \in$ r.intF. For otherwise, as before, using again Theorem18.2 from [R], we can conclude that $x_{o}$ sits in a face of $B_{E}$ strictly contained in $F$.

Let $l$ be the line passing through $x_{0}$ and $z$ and get $w$ in $l \cap F$ such that $x_{0}=\lambda z+(1-\lambda) w$ for some $\lambda, 0<\lambda<1$. Clearly $f_{i+1}\left(x_{0}\right)<f_{i+1}(z)$ now implies

$$
\inf _{y \in F \cap L} f_{i+1}(y) \leq f_{i+1}(w)<f_{i+1}\left(x_{0}\right)
$$


and this completes the proof.

We can now state and prove a technical but usable characterization of strongly proximinal subspaces of finite codimension.

Theorem 2.5. Let $X$ be a normed linear space and $Y$ be a proximinal subspace of finite codimension $n$ in $X$. Then $Y$ is strongly proximinal if and only if for every basis $f_{1}, \cdots, f_{n}$ of $Y^{\perp}$

$$
\lim _{\epsilon \rightarrow 0}\left[\sup \left\{d\left(x, J_{X}\left(f_{1}, \cdots, f_{i}\right): x \in J_{X}\left(f_{1}, \cdots, f_{i}, \epsilon\right)\right\}\right]=0\right.
$$

for $1 \leq i \leq n$.

Proof. We first prove necessity. Consider any basis $f_{1}, \cdots, f_{n}$ of $Y^{\perp}$. Fix $i \in\{1,2, \cdots, n\}$ and let $\eta>0$ be given. It suffices to show that there exists $\epsilon>0$ such that

$$
y \in J_{X}\left(f_{1}, \cdots, f_{i}, \epsilon\right) \Rightarrow \exists z \in J_{X}\left(f_{1}, \cdots, f_{i}\right) \text { with }\|y-z\|<\eta .
$$

By condition 4) of Proposition 2.3, given $x \in X$ with $\|x\|=1=d(x, Y)$ and $\eta>0$ there exists $\delta>0$ such that

$$
\begin{aligned}
& y \in B_{X},\|x-y+Y\|<\delta \Rightarrow \exists z \in B_{X} \text { with }\|y-z\|<\eta \\
& \text { and } x-z \in Y .
\end{aligned}
$$

Since $Y$ is of finite codimension, given $\delta>0$ we can get $\epsilon>0$ by a compactness argument, so that

$$
y \in J_{X}\left(f_{1}, \cdots, f_{i}, \epsilon\right) \Rightarrow d\left(y+Y, J_{X}\left(f_{1}, \cdots, f_{i}\right)+Y\right)<\delta
$$

that is,

$$
y \in J_{X}\left(f_{1}, \cdots, f_{i}, \epsilon\right) \Rightarrow \exists x \in J_{X}\left(f_{1}, \cdots, f_{i}\right) \text { with }\|x-y+Y\|<\delta .
$$

It is easily verified that $\|x\|=d(x, Y)=1$. Hence, by (7), there exists $z \in B_{X}$ with $x-z \in Y$ and $\|y-z\|<\eta$. Clearly $z \in J_{X}\left(f_{1}, \cdots, f_{i}\right)$ and this completes the proof for the necessity.

We now prove that the condition is sufficient. Choose any $x_{0}$ with

$$
\left\|x_{0}\right\|=d\left(x_{0}, Y\right)=1
$$


We prove that condition 3) of Proposition 2.3 holds for $x_{0}$.

We first assume that $\left.x_{0}\right|_{Y^{\perp}} \in \operatorname{ext}\left(S_{\left(Y^{\perp}\right)^{*}}\right)$. Then there exists a basis $f_{1}, \cdots, f_{n}$ of $Y^{\perp}$ such that $x_{0} \in J_{X}\left(f_{1}, \cdots, f_{n}\right)$, hence

$$
\left\{\left.x_{0}\right|_{Y^{\perp}}\right\}=\left.J_{X}\left(f_{1}, \cdots, f_{n}\right)\right|_{Y^{\perp}} .
$$

Now if $y \in B_{X}$ satisfies, for some $\epsilon>0$ and all $i \leq n$

$$
\left|f_{i}(y)-f_{i}\left(x_{0}\right)\right|<\epsilon
$$

then clearly $y \in J_{X}\left(f_{1}, \cdots, f_{n}, \epsilon\right)$. By assumption, given $\eta>0$, choosing $\epsilon$ small enough we can get $z \in J_{X}\left(f_{1}, \cdots, f_{n}\right)$ with $\|y-z\|<\eta$, hence $z \in B_{X} \cap(x+Y)$.

If $\left.x_{0}\right|_{Y^{\perp}} \notin \operatorname{ext}\left(S_{\left(Y^{\perp}\right)^{*}}\right)$, by Proposition 2.4, there is a basis $f_{1}, \cdots, f_{n}$ of $Y^{\perp}$ such that $x_{0} \in J_{X}\left(f_{1}, \cdots, f_{l}\right), l<n$, and the inequalities given by (6) hold. Now if $y \in B_{X}$ satisfies (8), we first approximate $y$ by a $z \in J_{X}\left(f_{1}, \cdots, f_{l}\right)$ and at the second step we proceed by induction to "shift" this $z$ and fix the equations $f_{i}\left(z^{\prime}\right)=f_{i}\left(x_{0}\right)$ for $l+1 \leq i \leq n$.

Without loss of generality we assume that $\left\|f_{i}\right\|=1$ for $1 \leq i \leq n$. For $l+1 \leq i \leq n$ set

$$
\begin{array}{r}
\alpha_{i}=\sup \left\{f_{i}(x): f_{j}\left(x_{0}\right)=f_{j}(x) \text { for } 1 \leq j \leq i-1\right\}- \\
\inf \left\{f_{i}(x): f_{j}\left(x_{0}\right)=f_{j}(x) \text { for } 1 \leq j \leq i-1\right\} .
\end{array}
$$

Select $\alpha>0$ such that

$$
2 \alpha<\min \left\{\alpha_{i}: l+1 \leq i \leq n\right\} .
$$

Choose any $\epsilon>0$ and $\eta>0$ satisfying $\eta<\min \left\{\frac{\epsilon}{2 n}, \frac{\alpha \epsilon}{8 n}, \frac{\alpha}{2}\right\}$. By assumption, we can get $\delta, 0<\delta<\frac{\eta}{2}$ so that if $y \in B_{X}$ satisfies (8) with $\delta$, there exists $z \in J_{X}\left(f_{1}, \cdots, f_{l}\right)$ satisfying $\|y-z\|<\eta$. We have

$$
\begin{aligned}
\left|f_{l+1}\left(z-x_{0}\right)\right| & \leq\left|f_{l+1}(z-y)\right|+\left|f_{l+1}\left(y-x_{0}\right)\right| \\
& <\eta+\delta<2 \eta<\alpha
\end{aligned}
$$

It is clear from the above inequality that we can select $w \in J_{X}\left(f_{1}, \cdots, f_{l}\right)$ so that $\left|f_{l+1}(w-z)\right|>\alpha$ and either

$$
f_{l+1}(w)<f_{l+1}\left(x_{0}\right) \leq f_{l+1}(z)
$$


or

$$
f_{l+1}(w)>f_{l+1}\left(x_{0}\right) \geq f_{l+1}(z) .
$$

Therefore there exists a suitable convex combination $z_{1}=\lambda z+(1-\lambda) w$ so that $f_{l+1}\left(z_{1}\right)=f_{l+1}\left(x_{0}\right)$. We have

$$
2 \eta>\left|f_{l+1}\left(x_{0}-z\right)\right|=\left|f_{l+1}\left(z_{1}-z\right)\right|=(1-\lambda)\left|f_{l+1}(w-z)\right|
$$

and so

$$
1-\lambda<\frac{2 \eta}{\left|f_{l+1}(w-z)\right|}<\frac{2 \eta}{\alpha}<\frac{\epsilon}{4 n}
$$

Now, $z, w$ and $x_{0}$ are in $J_{X}\left(f_{1}, \cdots, f_{l}\right)$ and so

$$
f_{i}\left(z_{1}\right)=f_{i}\left(x_{0}\right) \text { for } 1 \leq i \leq l+1 .
$$

Also, using (9) we have,

$$
\begin{aligned}
\left\|y-z_{1}\right\| & \leq\|y-z\|+\left\|z-z_{1}\right\| \\
& <\eta+(1-\lambda)\|w-z\| \\
& \leq \eta+2(1-\lambda) \\
& <\frac{\epsilon}{2 n}+\frac{\epsilon}{2 n} \\
& <\frac{\epsilon}{n}
\end{aligned}
$$

Now we repeat the arguement with $z$ replaced by $z_{1}$ and the set $J_{X}\left(f_{1}, \cdots, f_{l}\right)$ replaced by the set $\left\{x \in X: f_{j}(x)=f_{j}\left(x_{0}\right), 1 \leq j \leq\right.$ $l+1\}$. Proceeding thus inductively, we get $z^{\prime}=z_{n-l} \in S_{X}$ satisfying

$$
f_{i}\left(z^{\prime}\right)=f_{i}\left(x_{0}\right) \text { for } 1 \leq i \leq n \text { and }\left\|y-z^{\prime}\right\|<\epsilon
$$

and this concludes the proof of the theorem.

Considering the special case when the subspace of $X$ is a hyperplane, we have

Proposition 2.6. Let $X$ be a Banach space. The following statements are equivalent.

1. Every proximinal hyperplane is strongly proximinal.

2. $N A_{1}=\left\{f \in S_{X^{*}}:\|\cdot\|_{X^{*}}\right.$ is $S S D$ at $\left.f\right\}$ 
Proof. This follows easily from Lemma 1.1 and Theorem 2.5.

Using Proposition 2.6. it is easy to construct a reflexive space with an hyperplane which is not strongly proximinal, by constructing a dual norm which is not everywhere $S S D$. Note however that when $X$ is reflexive, the set of hyperplanes which are not strongly proximinal is always "meager", since the dual norm is Fréchet differentiable (and thus SSD) outside a meager set. On the other hand, there are separable spaces which contain no strongly proximinal hyperplane. Such an example is provided by the natural predual of the Hardy space $H^{1}(D)$, since the natural norm of $H^{1}(D)$ is nowhere $S S D$, as follows from ([D-G-Z], Prop. III.4.5).

\section{Quasi-polyhedral points in dual spaces}

In [A-D], Amir and Deutsch defined the notion of quasipolyhedral point ((QP)-point). Wegmann showed [W] that their notion is equivalent to the one given below. We refer to $[\mathrm{D}-\mathrm{P}]$ and references therein for definitions and basic properties of polyhedral spaces.

Definition 3.1. Let $X$ be a Banach space and $x \in S_{X}$. Then $x$ is a quasipolyhedral point ((QP)-point) if there is $\alpha>0$ such that $J_{X^{*}}(y) \subseteq$ $J_{X^{*}}(x)$ for every $y \in S_{X} \cap B(x, \alpha)$.

Remark 3.2. Assume there exists a convex, open subset $C$ of a Banach space $X, x \in C \cap S_{X}$ and $D$ dense in $C \cap S_{X}$, such that $J_{X^{*}}(x) \cap J_{X^{*}}(y) \neq$ $\emptyset$ for every $y \in D$. Then $x$ is a (QP)-point.

Indeed, observe that

$$
\begin{aligned}
J_{X^{*}}(x) \cap J_{X^{*}}(y) \neq \emptyset \quad \forall y \in D \cap C & \Rightarrow\|x+y\|=2 \quad \forall y \in D \cap C \\
& \Rightarrow\|x+y\|=2 \quad \forall y \in S_{X} \cap C .
\end{aligned}
$$

We select $\alpha>0$ such that $B(x, 2 \alpha) \subseteq C$. If $y \in S_{X} \cap B(x, \alpha)$ then considering the 2- dimensional subspace generated by $x$ and $y$, and using the above implications, we see that there exists $z \in S_{X} \cap C$ so that $y$ is an interior point of the line segment joining $x$ and $y$. It is now clear that $J_{X^{*}}(y) \subseteq J_{X^{*}}(x)$.

Below we give an easy but useful connection between (QP)-points and strong sub-differentiability. Note that the converse implication in Lemma 3.3 below is clearly false; however, every point of a $S S D$ norm 
with the property $\beta$ of Lindenstrauss is quasi-polyhedral ([G-JS-M], Prop.3.2).

Lemma 3.3. Let $X$ be a Banach space and $x \in S_{X}$ be a (QP)-point. Then the norm of $X$ is SSD at $x$.

Proof. We recall that the norm is $S S D$ at $x$ if

$$
\lim _{t \rightarrow 0^{+}} \frac{1}{t}[\|x+t h\|-\|x\|]
$$

is uniform in $h \in S_{X}$. If $x$ is a (QP)-point, then there exists $\alpha>0$ such that if $y \in B(x, 2 \alpha) \cap S_{X}$ then $J_{X}(y) \subseteq J_{X}(x)$. For any $h \in S_{X}$ and $t \in(0, \alpha)$, we have $\|x-w\|<2 \alpha$ where $w=\frac{x+t h}{\|x+t h\|}$. Thus $J_{X}(x+t h)=J_{X}(w) \subseteq J_{X}(x)$ which implies that $\|\cdot\|_{X}$ is linear over the line segment joining $x$ and $x+t h$. Clearly in this case we have

$$
\frac{1}{t}[\|x+t h\|-\|x\|]=\lim _{t \rightarrow 0^{+}} \frac{1}{t}[\|x+t h\|-\|x\|] .
$$

and hence the lemma.

We can now state and prove the main result of this paper. Let us recall that the if $Y$ is a finite codimensional subspace of a Banach space $X$, the condition " $Y$ is proximinal in $X$ " is in full generality strictly weaker than (b) and strictly stronger than (a) below (see [I2]).

Theorem 3.4. Let $X$ be a Banach space such that every $f \in N A_{1}$ is a (QP)-point of $\|.\|_{X^{*}}$. If $Y$ is a closed, finite codimensional subspace of $X$ then the following are equivalent:

(a) $Y^{\perp} \subseteq N A$.

(b) $Y$ is strongly proximinal in $X$.

Proof. If (a) holds, the annihilator space $Y^{\perp}$ is polyhedral by Lemma 1 of [G-I]. Moreover, it follows from the proof of Theorem 3 in [G-I] that $Y$ is proximinal in $X$. Now since every $f \in S_{Y^{\perp}}$ is a (QP)-point, by Lemma 3.3, \|\|$_{X^{*}}$ is SSD at every point of $S_{Y^{\perp}}$ and hence at every point of $Y^{\perp}$. Therefore condition 2) of Lemma 1.1 holds.

Since $Y^{\perp}$ is polyhedral, it follows from ([W], Theorem 4.4) and an easy induction that given any basis $f_{1}, \cdots, f_{n}$ of $Y^{\perp}$ and $i, 1 \leq i \leq n$, we can get appropriate scalars $\lambda_{j}>0$ for $1 \leq j \leq i$ so that

$$
J_{X}\left(f_{1}, \cdots, f_{i}\right)=J_{X}\left(\sum_{j=1}^{i} \lambda_{j} f_{j}\right)
$$


Hence, given $\epsilon>0$, we can easily get $\epsilon^{\prime}>0$ such that

$$
J_{X}\left(f_{1}, \cdots, f_{i}, \epsilon^{\prime}\right) \subseteq J_{X}\left(\sum_{j=1}^{i} \lambda_{j} f_{j}, \epsilon\right)
$$

Now condition 2) of lemma 1.1 shows that we can apply Theorem 2.5 and conclude to the strong proximinality of $Y$.

Conversely if $Y$ is strongly proximinal, $Y$ is proximinal. As $Y$ is of finite codimension, $Y^{\perp} \subseteq N A$ by a characterization of proximinal subspaces of finite codimension given in [G]. This concludes the proof.

Examples 3.5. Fonf and Lindenstrauss showed in [F-L] that if a Banach space has a boundary $B$ such that $B^{\prime} \cap N A_{1}=\emptyset$ then every $f \in N A_{1}$ is a (QP)-point. This property is hereditary and it is satisfied in particular by every polyhedral Lindenstrauss space [G-M]. In fact, it is shown in $[\mathrm{F}-\mathrm{L}]$ that if $X$ is a separable Banach space the following facts are equivalent:

1. There exists a boundary $B$ such that $B^{\prime} \cap N A_{1}=\emptyset$.

2. For any sequence $\left(f_{n}\right) \subseteq N A_{1}$ converging weak* to $f \in N A_{1}$ there exists a positive integer $N$ such that $J_{X}\left(f_{n}\right) \cap J_{X}(f) \neq \emptyset$ for $n \geq N$.

3. Same as (2) with $J_{X}\left(f_{n}\right) \subseteq J_{X}(f)$ for $n \geq N$.

We refer to Remark 3.2. for the derivation of the (QP)-property from the above condition 2), even when weak-star convergence is replaced by convergence in norm.

A special case (considered in $[\mathrm{G}-\mathrm{I}]$ ) of the above is when $X$ is a subspace of $c_{0}(\mathbf{N})$.

\section{Remarks 3.6.}

1. As mentioned above, the Fonf-Lindenstrauss condition is hereditary. However, if $Y$ is a closed subspace of a Banach space $X$ and $g \in N A_{1}(Y)$, assuming that every norm preserving extension $f \in B_{X^{*}}$ of $g$ is a (QP)-point does not imply in general that $\|\cdot\|_{Y^{*}}$ is $\mathrm{SSD}$ at $g$. Here is an example:

Take $Y$ such that $\|$. $\|_{Y^{*}}$ is Gateaux smooth on $Y^{*} \backslash\{0\}$ and nowhere Frechet smooth (e.g. $Y=$ the natural predual of the 
Hardy space $H^{1}(D)$; see [D-G-Z], Prop.III.4.5). Then $\|\cdot\|_{Y^{*}}$ is nowhere SSD except at $\{0\}$. Put $Y \subseteq X=C\left(\left(B_{Y^{*}}, w^{*}\right)\right)$. Pick a weak* exposed point $g$ of $B_{Y^{*}}$. Then:

a) The Dirac measure $\delta_{g}$ at $g$ is the only $f \in B_{X^{*}}$ such that $\left.f\right|_{Y}=g$. Indeed, if $y$ in $S_{Y}$ exposes $g$, then $|h(y)|<1$ for all $h \in B_{Y^{*}}$. It is now clear that any measure other than $\delta_{g}$ in $S_{(C(K))^{*}}$, where $K=\left(B_{Y^{*}}, w^{*}\right)$, takes value strictly less than 1 at $y$.

b) It is easy to see that the measure $\delta_{g}$ is a (QP)-point of $S_{X^{*}}$.

However $\|.\|_{Y^{*}}$ is not SSD at $g$ and this together with a) and b) proves the claim.

2. For a given $f \in N A_{1}$, condition b) in Example 3.5 is in general stronger than the corresponding property with $\|$. \| convergence. For instance, set $\hat{\mathbb{N}}=\mathbb{N} \cup\{\infty\}, X=\left(C\left(\hat{\mathbb{N}},\|\cdot\|_{\infty}\right), f=\delta_{\infty}\right.$ and $f_{n}=(1-1 / n) \delta_{n}-1 / n \delta_{\infty}$. Clearly, $f \in N A_{1},\left(f_{n}\right) \subseteq N A_{1}, \quad\left(f_{n}\right)$ converges weak ${ }^{*}$ to $f$ and $J_{X^{* *}}\left(f_{n}\right) \cap J_{X^{* *}}(f)=\emptyset$ for all $n$. However $\delta_{\infty}$ is a $(\mathrm{QP})$-point of $X^{*}$.

3. Fonf and Lindenstrauss [F-L] have also shown: 1) there exists a polyhedral Banach space containing a 2-codimensional proximinal subspace $Y$ (in particular $Y^{\perp} \subseteq N A$ ) such that $Y^{\perp}$ is not polyhedral. 2) there exists a polyhedral space which contains a 2-codimensional subspace $Y$ such that $Y^{\perp} \subset N A$ but $Y$ is not proximinal.

\section{Selections of the metric projection}

In this section we show that the metric projection onto a closed proximinal subspace $Y$ of finite codimension, with $Y^{\perp}$ polyhedral, has a continuous selection. Before proving this result, we gather some observations about metric projections onto strongly proximinal subspaces.

Lemma 4.1. Let $Y$ be a strongly proximinal subspace of a normed linear space $X$. Then the metric projection $P_{Y}$ is upper semi-continuous.

Proof. This follows easily from the definition of strong proximinality and the triangle inequality. 
Proposition 4.2. Let $X$ be a normed linear space and $Y$ be a strongly proximinal subspace of $X$. Then the metric projection $P_{Y}$ onto $Y$ has a first Baire class selector.

Proof. By Lemma 4.1 above, the set valued map $P_{Y}$ is upper semicontinuous and by Jayne-Rogers selection theorem ([J-R]), $P_{Y}$ has a first Baire class selector.

Remark 4.3. A finite dimensional subspace is always strongly proximinal as can easily be seen with a compactness argument. However, continuous selections are not available in general for metric projections onto finite dimensional subspaces. A.L.Brown $[\mathrm{B}]$ has contructed an interesting counterexample of a 3 -dimensional space that contains a one dimensional subspace whose metric projection has no continuous selection.

The following simple lemma is a special case of a much more general result $([\mathrm{D}])$ on "stable" convex sets.

Lemma 4.3. Let $E$ be a finite dimensional polyhedral space and let $\operatorname{ext}\left(B_{E}\right)=\left\{e_{1}, \cdots, e_{k}\right\}$. Then there exists a continuous map $\phi: B_{E} \rightarrow$ $\left(\mathbb{R}^{+}\right)^{k}$ such that

$$
\phi(x)=\left(\lambda_{i}(x)\right)_{1 \leq i \leq k}
$$

where

$$
\sum_{i} \lambda_{i}(x)=1, x=\sum_{i=1}^{k} \lambda_{i}(x) e_{i}
$$

for all $x \in B_{E}$.

Remark 4.4. It is well-known that there may not be any continuous selection of a representing measure supported by extreme points, even in 3 -dimensional spaces. An example is provided by the space $\mathbb{R}^{3}$ equipped with the norm whose unit ball is the convex hull of

$$
\left\{(x, y, 0): x^{2}+y^{2}=1\right\} \cup\{( \pm 1,0, \pm 1)\} .
$$

We can now prove

Proposition 4.5. Let $X$ be a Banach space and $Y$ be a closed proximinal subspace of finite codimension in $X$ such that $Y^{\perp}$ is polyhedral. Then the metric projection $P_{Y}$ onto $Y$ has a continuous selection. 
Proof. Recall that for $x \in X$ with $d(x, Y)=1$,

$$
Q_{Y}(x)=x-P_{Y}(x)=\left\{z \in X:\|z\|=1, f(z)=f(x) \forall f \in Y^{\perp}\right\}
$$

Let $\operatorname{ext}\left(B_{\left(Y^{\perp}\right)^{*}}\right)=\left\{e_{1}, \cdots, e_{k}\right\}$. Pick $x_{i} \in B_{X}$ such that $\left.x_{i}\right|_{Y^{\perp}}=e_{i}$. Consider any $x \in X$ with $d(x, Y)=1$ and choose continuously, as in Lemma 4.3, positive scalars

$$
\mu_{i}(x)=\lambda_{i}\left(\left.x\right|_{Y^{\perp}}\right) \text { for } 1 \leq i \leq k
$$

so that

$$
\sum_{i=1}^{k} \mu_{i}(x)=1 \text { and }\left.x\right|_{Y^{\perp}}=\sum_{i=1}^{k} \mu_{i}(x) e_{i} .
$$

Fact. $\sum_{i=1}^{k} \mu_{i}(x) Q_{Y}\left(x_{i}\right) \subseteq Q_{Y}(x)$.

We have

$$
Q_{Y}\left(x_{i}\right)=\left\{z \in X:\|z\|=1, f(z)=f\left(x_{i}\right) \forall f \in Y^{\perp}\right\}
$$

Now

$$
t \in \sum_{i=1}^{k} \mu_{i}(x) Q_{Y}\left(x_{i}\right) \Rightarrow t=\sum_{i=1}^{k} \mu_{i}(x) z_{i}
$$

where $z_{i} \in Q_{Y}\left(x_{i}\right)$ for $1 \leq i \leq k$. By (10) we have for $f \in Y^{\perp}$,

$$
\begin{aligned}
f(t) & =\sum_{i=1}^{k} \mu_{i}(x) f\left(z_{i}\right)=\sum_{i=1}^{k} \mu_{i}(x) f\left(x_{i}\right) \\
& =f\left(\sum_{i=1}^{k} \mu_{i}(x) x_{i}\right)=f(x) .
\end{aligned}
$$

Moreover, $\|t\| \leq 1$, hence $t \in Q_{Y}(x)$.

Now the map $x \rightarrow\left(\mu_{i}(x)\right)_{i=1}^{k}$ is continuous. Hence the set valued map

$$
x \rightarrow \sum_{i=1}^{k} \mu_{i}(x) Q_{Y}\left(x_{i}\right)
$$

is continuous (hence in particular lower semi-continuous) on the domain

$$
\{x \in X: d(x, Y)=1\}
$$


and so is

$$
T_{Y}(x)=x-\sum_{i=1}^{k} \mu_{i}(x) Q_{Y}\left(x_{i}\right) \subseteq P_{Y}(x)
$$

We now extend $T_{Y}$ to whole of $X$ by scaling and clearly this extension is continuous on $X$. Finally Michael's selection theorem ([M]) provides a continuous selection for $T_{Y}$ and hence for $P_{Y}$.

Examples 4.6. Let $X$ be a Banach space such that every norm attaining functional in $S_{X^{*}}$ is a $(\mathrm{QP})$-point of $S_{X^{*}}$. If $Y$ is a finite codimensional subspace of $X$, then by Theorem 3.5. $Y$ is proximinal if and only if $Y^{\perp}$ is contained in $N A$, if and only if $Y$ is strongly proximinal. Moreover, by the proof of Theorem 3.5, $Y^{\perp}$ is polyhedral when these conditions hold. Now by the above proposition, the metric projection $P_{Y}$ has a continuous selection.

By [F-L], this applies in particular when $X$ is a separable Banach space such that there exists a boundary $B$ for $X$ with $B^{\prime} \cap N A_{1}=\emptyset$.

Acknowledgement. This work was completed while the first named author was visiting the University of Pondicherry in January 1999. He is glad to express his thanks to this Institution for its warm hospitality and support. The financial support of the National Board for Higher Mathematics is also gratefully acknowledged.

\section{References}

[A-D] D. Amir, F. Deutsch: Suns, moons and quasi-polyhedra, J. Approx. Theory, 6 (1972), 176-201.

[B] A.L. Brown: Some problems in linear Analysis, Ph.D dissertation, Cambridge University, 1961.

[D] G. Debs: Applications affines ouvertes et convexes compacts stables, Bull. Sc. Math. 2e série, 102 (1978), 401-414.

[D-G-Z] R. Deville, G. Godefroy, V. Zizler: Smoothness and renormings in Banach spaces, Pitman Monographs 64, Longman ed. (1993).

[D-P] R. Durier, P. L. Papini: Polyhedral norms in an infinite dimensional space, Rocky Mountain J. of Math., 23, 3 (1993), 863-876.

[F-L] V. Fonf, J. Lindenstrauss: On quotients of polyhedral spaces, Preprint. 
[F-P] C. Franchetti, R. Paya: Banach spaces with strongly subdifferentiable norm, Boll. Uni. Mat. Ital. VII.B(1993), 45-70

[G] A.L. Garkavi: On the best approximation by elements of infinite dimensional subspaces of a certain class, Mat. Sb .62, (1963),104-120.

[G-M] A. Gleit, R. MacGuigan: A note on polyhedral Banach spaces, Proc. Amer. Math. Soc. 33 (1972), 398-404.

[G-M-Z] G. Godefroy, V. Montesinos, V. Zizler: Strong subdifferentiability of norms and geometry of Banach spaces, Comment. Math. Univ. Carolinae 36,3 (1995), 493-502.

[G-I] G. Godefroy, V. Indumathi: Proximinality in subspaces of $c_{0}(\mathbf{N}), J$. Approx. Theory, 101 (1999), 175-181.

[G-JS-M] A. S. Granero, M. Jimenez-Sevilla, J. P. Moreno: Geometry of Banach spaces with property $\beta$, Israel J. Math., 111 (1999), 263-273.

[I] V. Indumathi: Proximinal subspaces of finite codimension in general normed linear spaces, Proc. London Math. Soc. (3), 45 (1982), 435455 .

[I2] V. Indumathi: On transitivity of proximinality, J. of Approx. Theory, 49,2 (1987), 130-143.

[J-R] J. Jayne, C. A. Rogers: Borel selectors for upper semicontinuous set valued maps, Acta Math. 155 (1985), 41-79.

[K] Ka Sing Lau: On a sufficient condition of proximity, Trans. Amer. Math. Soc, 251 (1979) 343-356.

[L-T] J. Lindenstrauss, L. Tzafriri: Classical Banach spaces, vol.1, sequences spaces, Springer-Verlag, 1977.

[M] E. Michael: Continuous selections I, Annals of Maths. 63 (1956), 361382 .

[R] R.T. Rockafellar: Convex Analysis, Princeton N.J. 1970.

[W] R. Wegmann: Some properties of the peak-set-mapping, J. Approx. Theory, 8(1973) 262-284. 
Gilles Godefroy

Equipe d'Analyse

Université Paris 6, boite 186

4, Place Jussieu

75252 Paris Cedex 05

France

E-mail: gig@ccr.jussieu.fr

V. Indumathi

Department of Mathematics

University of Pondicherry

Kalapet

Pondicherry 605014

India

Recibido: 10 de Enero de 2000

E-mail: indumath@md2.vsnl.net.in
Revisado: 24 de Octubre de 2000 